PROCEEDINGS OF THE

AMERICAN MATHEMATICAL SOCIETY

Volume 125, Number 4, April 1997, Pages 1203-1208

S 0002-9939(97)03624-1

\title{
RICCI SOLITONS ON COMPACT KÄHLER SURFACES
}

\author{
THOMAS IVEY
}

(Communicated by Peter Li)

\begin{abstract}
We classify the Kähler metrics on compact manifolds of complex dimension two that are solitons for the constant-volume Ricci flow, assuming that the curvature is slightly more positive than that of the single known example of a soliton in this dimension.
\end{abstract}

\section{INTRODUCTION}

The Ricci flow $\partial g / \partial t=-2 \operatorname{Ric}(g)$ is an evolution equation for Riemannian metrics. In several cases [H1], [H2], [H3] the flow converges, up to re-scaling, to a metric of constant curvature. However, "soliton" solutions to the flow give examples where the Ricci flow does not uniformize the metric, but only changes it by diffeomorphisms. In this paper we are concerned with soliton solutions for the constant-volume Ricci flow

$$
\partial g / \partial t=-2 \operatorname{Ric}(g)+2 \frac{r}{n} g
$$

on a compact manifold. (Here, $r$ is the average of the scalar curvature.) Soliton solutions will be generated by initial data satisfying

$$
\mathcal{L}_{X} g=-2 \operatorname{Ric}(g)+2 \frac{r}{n} g
$$

for some vector field $X$. We will refer to these as compact solitons.

In dimensions two [H3] and three [I2] the only compact solitons are constant curvature metrics, which are fixed under the constant volume flow. Nontrivial examples of compact solitons were constructed by Koiso [K], in the form of Kähler metrics of cohomogeneity one on certain $\mathbb{P}^{1}$ bundles on $\mathbb{C P}^{n}$. (For an elegant presentation of this construction, see $[\mathrm{C}]$.) These examples start in dimension four, with a metric on $\mathbb{P}(L \oplus \mathbb{C})$, where $L$ is the hyperplane bundle on $\mathbb{C P}^{1}$. We will discuss this particular example in more detail below.

In this paper we show that a nontrivial soliton that is a Kähler metric on a compact complex surface has curvature at least as negative as Koiso's example. (It is easy to see from applying the maximum principle to the evolution equation for the scalar curvature under the Ricci flow that any compact soliton must at least have strictly positive scalar curvature, or else it is an Einstein metric [I2].)

Received by the editors July 26, 1995 and, in revised form, October 24, 1995.

1991 Mathematics Subject Classification. Primary 53C20, 53C21.

Key words and phrases. Ricci flow, solitons, Kähler surfaces.

(C)1997 American Mathematical Society 
Main Theorem. Suppose $M^{4}$ is a compact complex surface and $g$ is a Kähler metric satisfying (1). If the sum $a_{1}+a_{2}$ of the lowest eigenvalues of the restriction of the curvature operator of $g$ to the space $\Lambda_{-}^{2}$ of anti-self-dual forms ${ }^{1}$ is nonnegative throughout $M$, then either $M$ is $S^{2} \times S^{2}$ with $g$ a product of metrics of the same constant positive curvature, or $M$ is $\mathbb{C P}^{2}$ with $g$ the Fubini-Study metric.

In $\S 1$ of this paper we discuss the curvature operator for a Kähler surface, and the curvature of the Koiso example. In $\S 2$ we show how the soliton condition implies positivity of the curvature, proving our theorem.

That $M$ must be $S^{2} \times S^{2}$ or $\mathbb{C P}^{2}$ also follows from the results of Micallef and Wang $[\mathrm{MW}]$, since our hypothesis is that $g$ is a Ricci soliton Kähler metric with nonnegative isotropic curvature (see [H5] for details). The results in this paper differ in that we are able to get much more information about the curvature of $g$ using the soliton assumption.

\section{KÄHLER CURVATURE}

Recall that the curvature operator of a Riemannian metric is the Riemann curvature tensor regarded as a symmetric endomorphism of $\Lambda^{2} T M$, or of $\Lambda^{2} T^{*} M$ by metric duality. On a Kähler surface, the curvature operator has image in the holonomy algebra $\mathfrak{u}(2) \subset \mathfrak{s o}(4)$ spanned by the (1,1)-forms. If we choose an orthonormal frame $\omega^{1}, \omega^{2}, \omega^{3}, \omega^{4}$ so that $\omega^{1}+i \omega^{2}$ and $\omega^{3}+i \omega^{4}$ are $(1,0)$-forms, then the Kähler form $\Omega=\omega^{1} \wedge \omega^{2}+\omega^{3} \wedge \omega^{4}$ and the anti-self-dual forms $\psi_{1}=\omega^{1} \wedge \omega^{2}-\omega^{3} \wedge \omega^{4}$, $\psi_{2}=\omega^{2} \wedge \omega^{3}-\omega^{1} \wedge \omega^{4}$, and $\psi_{3}=\omega^{3} \wedge \omega^{1}-\omega^{2} \wedge \omega^{4}$ give a basis for the (1,1)-forms. When expressed using this basis, the curvature operator has the form

$$
\left(\begin{array}{cccccc}
a & 0 & 0 & b_{1} & b_{2} & b_{3} \\
0 & 0 & 0 & 0 & 0 & 0 \\
0 & 0 & 0 & 0 & 0 & 0 \\
b_{1} & 0 & 0 & & & \\
b_{2} & 0 & 0 & & A & \\
b_{3} & 0 & 0 & & &
\end{array}\right) .
$$

The 3 by 3 submatrices reflect the decomposition $\Lambda^{2} T^{*} M=\Lambda_{+}^{2} \oplus \Lambda_{-}^{2}$, and the zero rows and columns correspond to forms in $\Lambda_{+}^{2}$ that are orthogonal to the Kähler form. Because of the Bianchi identity, $a=\operatorname{tr}(A)$.

The holonomy group $U(2)$ acts on the curvature operator by the standard, but simultaneous, actions of $S O(3)$ on the symmetric matrix $A$ and vector $b=\left(b_{1}, b_{2}, b_{3}\right)$. Since the invariant $a$ is one-half the scalar curvature, any soliton must have $a>0$ at every point. However, other natural curvature conditions translate to conditions involving the simultaneous invariants of $A$ and $b$. Since the eigenvalues of the Ricci tensor are $a \pm|b|$, the Ricci curvature is positive if and only if $a>|b|$. Furthermore, a complex 2-plane $V \wedge J V$ is dual to a (1,1)-form $u \Omega+v_{1} \psi_{1}+v_{2} \psi_{2}+v_{3} \psi_{3}$ satisfying the decomposability condition $u^{2}=v_{1}^{2}+v_{2}^{2}+v_{3}^{2}$. Then, by scaling to get $u=1$, we see that the Kähler metric has positive holomorphic sectional curvature if and only if

$$
a+2 b \cdot v+{ }^{t} v A v>0
$$

\footnotetext{
${ }^{1}$ We adopt an orientation so that the Kähler form is self-dual.
} 
for all unit vectors $v$ in $\mathbb{R}^{3}$. Similarly, the metric has positive holomorphic bisectional curvature if and only if

$$
a+b \cdot v+b \cdot w+{ }^{t} v A w>0
$$

for all unit vectors $v$ and $w$.

Let $a_{1} \leq a_{2} \leq a_{3}$ be the eigenvalues of $A$. Then the hypothesis of the main theorem is that $a_{1}+a_{2} \geq 0$ everywhere on $M$. Koiso's example of a Kähler Ricci soliton doesn't satisfy this. The metric can be constructed as a $U(2)$-symmetric Kähler metric on $\mathbb{C}^{2}$, satisfying certain boundary conditions. If $\phi(t)$ is the derivative of the Kähler potential with respect to $t=\log \left(\left|z_{1}^{2}\right|+\left|z_{2}^{2}\right|\right)$, then the Ricci soliton condition becomes an ODE

$$
\frac{\phi^{\prime \prime}}{\phi^{\prime}}+\left(c_{1}+\phi^{-1}\right) \phi^{\prime}=2-\phi
$$

where $c_{1} \doteq-0.5276$ is dictated by the boundary conditions. (Our notation here comes from $[\mathrm{C}]$.) The curvature operator takes the form

$$
\left(\begin{array}{cccccc}
a & 0 & 0 & \mu & 0 & 0 \\
0 & 0 & 0 & 0 & 0 & 0 \\
0 & 0 & 0 & 0 & 0 & 0 \\
\mu & 0 & 0 & a-2 \lambda & 0 & 0 \\
0 & 0 & 0 & 0 & \lambda & 0 \\
0 & 0 & 0 & 0 & 0 & \lambda
\end{array}\right)
$$

where

$$
\lambda=\frac{2}{\phi^{2} \phi^{\prime}}\left(\left(\phi^{\prime}\right)^{2}-\phi \phi^{\prime \prime}\right)
$$

and

$$
a-\mu=4\left(\phi-\phi^{\prime}\right) / \phi^{2}, \quad a+\mu=2\left(\left(\phi^{\prime \prime}\right)^{2}-\phi^{\prime} \phi^{\prime \prime \prime}\right) /\left(\phi^{\prime}\right)^{3} .
$$

The boundary conditions give $\phi \searrow 1, \phi^{\prime} \rightarrow 0$ as $t \rightarrow-\infty$ and $\phi \nearrow 3, \phi^{\prime} \rightarrow 0$ as $t \rightarrow+\infty$. Plotting the solution of (2) reveals that $\lambda$ is negative near the $t=-\infty$ end, and hence $a_{1}+a_{2}$ is negative there. On the other hand, the Ricci curvature is strictly positive everywhere, reflecting the fact that the first Chern class of the manifold is positive.

\section{Coarse Classification of SOlitons}

In this section we will establish that if $A$ is 2-nonnegative (i.e., $a_{1}+a_{2} \geq 0$ ), then the soliton condition forces the entire curvature operator to be nonnegative. The argument uses Hamilton's version of the maximum principle for parabolic evolution equations on vector bundles [H2]. This says that if the ODE obtained by dropping the Laplacian preserves a convex set in the bundle, then the original evolution equation also preserves the set: sections that lie in the set at time zero continue to lie in the set. We will define such sets using inequalities for sections of $S^{2}\left(\Lambda^{2} T^{*} M\right)$, the bundle in which the curvature operator takes value. (As explained in [H2], the maximum principle is applied to the Ricci flow by carrying the curvature operator, by an evolving gauge transformation, to a copy $E$ of $S^{2}\left(\Lambda^{2} T^{*} M\right)$ with a fixed metric. The Levi-Civita connection induces an evolving connection on this copy, and our set is required to be parallel at all times. However, the connections are always Hermitian, and we will define sets in terms of the $U(2)$-invariants discussed above.) 
We should note that $a_{1}$ and $a_{1}+a_{2}$ are concave functions on the space of curvature operators (for example, $a_{1} \geq C$ defines a convex set), $|b|$ is a convex function, and $a$ is a linear function. As explained in [H2], the ODE for the curvature operator implies the following differential inequalities for these invariants:

$$
\begin{gathered}
d a_{1} / d t \geq a_{1}^{2}+2 a_{2} a_{3}, \\
d\left(a_{1}+a_{2}\right) / d t \geq a_{1}^{2}+a_{2}^{2}+2\left(a_{1}+a_{2}\right) a_{3}, \\
d|b| / d t \leq\left(a+a_{3}\right)|b|,
\end{gathered}
$$

along with the differential equation

$$
d a / d t=a^{2}+|b|^{2} .
$$

While these differential equations come from the unnormalized Ricci flow, we can still use them to prove scale-invariant results for the normalized Ricci flow, since solutions for the two flows, starting with the same metric, differ only by rescaling in space and time (see [H1]).

Lemma 2.1. If $R>0$ and $a_{1}+a_{2} \geq 0$ everywhere, then $a_{1} \geq 0$ everywhere.

Proof. Let $f(x)$ be a positive, increasing, concave function of $x$ such that $\lim _{x \rightarrow \infty} f(x) / x=0$ (e.g., take $f(x)=\log (x+1)$ ). We can scale $f$ to arrange that

$$
a_{1}+f(a) \geq 0
$$

holds everywhere on $M$ at time zero. This defines a parallel, convex set in $E$. Since $a_{2} \geq\left(a_{1}+a_{2}\right) / 2 \geq 0$, we see that $d a_{1} / d t \geq 0$. Then the set defined by (7) is preserved by the ODEs. Thus, (7) holds for the solution of the unnormalized Ricci flow for all $t \geq 0$.

Recall that $a=R / 2$. Since

$$
\frac{\partial R}{\partial t}=\Delta R+2|\operatorname{Ric}|^{2} \geq \Delta R+\frac{1}{2} R^{2},
$$

$\inf _{M} R \rightarrow \infty$ in finite time. Since $R_{\max } / R_{\min }$ is bounded on the soliton, this happens everywhere at once. Then (7) gives us a negative lower bound

$$
\frac{a_{1}}{a} \geq-\frac{f(a)}{a}
$$

that goes to zero everywhere. Since $a_{1} / a$ is scale-invariant, then for the solution of the normalized flow $\inf _{M} a_{1} / a$ must also become nonnegative as $t \rightarrow \infty$. Then since we assume the solution is a soliton, $a_{1}$ must have been nonnegative all along.

Lemma 2.2. If $a_{1} \geq 0$ everywhere, then the curvature operator of $M$ is nonnegative.

Proof. Let

$$
N=\left(\begin{array}{cccc}
a & b_{1} & b_{2} & b_{3} \\
b_{1} & & & \\
b_{2} & & A & \\
b_{3} & & &
\end{array}\right)
$$


and let $n_{1}$ be the least eigenvalue of $N$. (This will be invariant under the holonomy representation $S O(3) \subset S O(4)$.) It will suffice to prove that $n_{1} \geq 0$. The ODE corresponding to the evolution equation of $N$ is

$$
\frac{d N}{d t}=N^{2}+\left(\begin{array}{cc}
0 & 0 \\
0 & A^{\#}
\end{array}\right),
$$

where $A^{\#}$ is the adjoint of $A$. Since $A$ has nonnegative eigenvalues, the same is true of the r.h.s. of (8). This implies that $d n_{1} / d t \geq 0$.

Let $f$ be as in the previous lemma. Then we can scale $f$ to arrange that $n_{1}+$ $f(R) \geq 0$ at time zero. This defines a parallel, convex set in $E$ that is preserved under (8). We argue as before that $\inf _{M} n_{1} / R$ becomes nonnegative as time goes on, and so must have been nonnegative all along.

Proposition 2.3. $M$ is either $S^{2} \times S^{2}$ with a product of metrics of the same constant positive curvature, or $M$ is $\mathbb{C P}^{2}$ with a metric of positive holomorphic bisectional curvature.

Proof. According to Theorem 8.3 in [H2], the image of the curvature operator of $M$ is a fixed Lie subalgebra of $\mathfrak{s o}(4)$ of constant rank on $M$. Because $M$ is Kähler, the possibilities are limited to:

$\mathfrak{u}(2)$ : In this case, $N$ is positive definite. While the curvature operator is only nonnegative, its restriction to decomposables, giving the sectional curvature, is strictly positive. This implies that the holomorphic bisectional curvature is positive, since

$$
\operatorname{Rm}(X, J X, Y, J Y)=\operatorname{Rm}(X, Y, X, Y)+\operatorname{Rm}(X, J Y, X, J Y)
$$

by the Bianchi identity. It now follows by the solution of the Frankel conjecture - in fact, the case proved by Frankel $[\mathrm{F}]$ - that $M$ is biholomorphic to $\mathbb{C P}^{2}$.

Note that the Futaki functional for any Kähler metric on $\mathbb{C P}^{n}$ is zero. It follows by an argument of Koiso ([K], Prop. 1.3) that $g$ is a Kähler-Einstein metric. Then, because of the sectional curvature, a theorem of Berger (see [G]) implies that $g$ is the Fubini-Study metric.

$\mathfrak{s o}(2) \times \mathfrak{s o}(2)$ : The universal cover $\widetilde{M}$ is $S^{2} \times S^{2}$ with the product of positive curvature metrics on each. In fact, since the Ricci tensor of $M$ is positive definite, $M$ must be simply connected [G]. The unnormalized Ricci flow shrinks one or the other $S^{2}$ to a point in finite time, with the ratio of the maximum curvature to the minimum curvature going to one. Because we assume $M$ is a soliton, both $S^{2}$ 's have to have the same constant curvature.

$\mathfrak{s o}(2)$ : In this case, $\widetilde{M}$ is isometric to $S^{2} \times \mathbb{R}^{2}$ with a flat metric on one factor and a positive curvature metric on the other. Consider the evolution equation for the average scalar curvature $r$ of $M$ under the normalized flow:

$$
\frac{d}{d t} \int R \mu_{g}=-\int\left\langle\frac{\partial g}{\partial t}, \operatorname{Ric}-\frac{1}{2} R g\right\rangle \mu_{g}
$$

(Here we have fixed the volume to be one.) We can calculate the integrand on the right pointwise by diagonalizing the Ricci tensor. We obtain

$$
\left\langle 2 \frac{r}{n} g-2 \text { Ric }, \text { Ric }-\frac{R}{2} g\right\rangle=-r \frac{R}{4}
$$

and $d r / d t=r^{2} / 4$. Since this would mean $r \rightarrow \infty$ in finite time, we can rule out this case. 


\section{ACKNOWLEDGMENTS}

We would like to thank Richard Hamilton and Fangyang Zheng for stimulating and helpful discussions, and the referee for some enlightening comments.

\section{REFERENCES}

[C] H. D. Cao, Existence of gradient Ricci-Kähler solitons, preprint (1994).

[F] T. Frankel, Manifolds with positive curvature, Pacific J. Math. 11 (1961), 165-174. MR 23a: 600

[G] P. Gauduchon, Surfaces Kähleriennes dont la courbure admet certaines conditions de positivité, in "Géométrie riemannienne en dimension 4", Cedic, Fernand Nathan, Paris (1981). CMP 17:05

[H1] R. S. Hamilton, Three-manifolds with positive Ricci curvature, J. Diff. Geom. 17 (1982) 255-306. MR 84a:53050

[H2] - Four-manifolds with positive curvature operator, J. D. G. 24 (1986) 153-179. MR 87m:53055

[H3] - The Ricci flow on surfaces, Contemporary Mathematics 71 (1988) 237-262. MR 89i:53029

[H4] The formation of singularities in the Ricci flow, preprint (1994).

[H5] , Four-manifolds with positive isotropic curvature, Surveys in Differential Geometry, Vol. II, International Press, 1995.

[I1] T. Ivey, On solitons for the Ricci flow, Ph.D. thesis, Duke University (1992).

[I2] T. Ivey, Ricci solitons on compact three-manifolds, Differential Geometry and its Applications 3 (1993), 301-307. MR 94j:53048

[K] N. Koiso, On rotationally symmetric Hamilton's equation for Kähler-Einstein metrics, Adv. Studies in Pure Math. 18-I (1990), 327-337. MR 93d:53057

[MW] M. J. Micallef, McK. Y. Wang, Metrics with nonnegative isotropic curvature, Duke Math. J. 72 (1993), 649-672. MR 94k:53052

Department of Mathematics, Case Western Reserve University, Cleveland, Ohio 44106-7058

E-mail address: txi4@po.cwru.edu 